\title{
One-step nucleic acid amplification: the possible value in assessing sentinel lymph node metastasis during mastectomy
}

This article was published in the following Dove Press journal: Breast Cancer - Targets and Therapy

\author{
Alison E Hunter-Smith \\ Zenon Rayter \\ Breast Surgery Unit, Bristol Breast \\ Care Centre, North Bristol NHS \\ Trust, Southmead Hospital, Westbury- \\ on-Trym, Bristol, UK
}

\begin{abstract}
Breast cancer is the most common cancer in women, worldwide, and 1,400 deaths per day are attributed to it. The success of national screening programs has seen breast cancers being diagnosed at an earlier stage. With conservative surgery to the breast demonstrating equivalent long-term outcomes, the last 10 years have seen a growing interest in the safety of less invasive management for the axilla in breast cancer patients. One-step nucleic acid amplification (OSNA) is a validated, reliable, and efficient tool in identifying micro- and macro-metastases intraoperatively. It is the most widely used intraoperative analysis tool within the United Kingdom, and is employed by over 320 units across Europe and Asia. Recent evidence from the AMAROS, IBCSG 23-01, and ACOSOG Z0011 trials has changed surgical practice in managing the axilla of patients with breast cancer. We propose a clinical algorithm demonstrating the role of OSNA as an intraoperative analysis tool in today's management of breast cancer as well as prospects for the future use of OSNA.
\end{abstract}

Keywords: breast cancer, sentinel lymph node, intraoperative assessment, one-stop nucleic acid amplification, mastectomy

\section{Breast cancer overview}

Breast cancer remains the most common cancer in women worldwide, with an estimated 1.68 million women diagnosed in 2012. ${ }^{1}$ In the United Kingdom, 55,222 new cases of breast cancer were diagnosed in 2014 - an increase of 1,883 cases compared to 2013.2,3 Mortality from breast cancer in 2012, worldwide, was reported as approximately 522,000 women; almost all those who die from breast cancer do so as a result of distant metastasis.

The roll-out of National Screening Programs over the past 30 years has increased early breast cancer diagnoses. Screen-detected invasive breast cancer tumors are smaller, of lower grade, are less likely to be node positive, and have better NPI scores than those breast cancer tumors presenting symptomatically. ${ }^{43}$

Following on from advances within breast conservation surgery, where long-term oncological outcomes are equivalent to radical surgical techniques, attention over the last decade turned to exploring less invasive axillary surgery and highlighted the importance of intraoperative sentinel node analysis, to accurately stage and treat patients with breast cancer.

\section{History of axillary surgery}

The concept of breast cancer being a local disease that first spreads to lymph nodes and, then, systemically was first described in 1757 when Henry LeDran founded the 
theory that surgery had the ability to cure breast cancer if recognized early enough. Accepting this notion, the era of large en bloc operations for breast cancer treatment began. Bernard Peyrilhe advised the removal of the breast along with all axillary contents and the pectoralis muscle in 1773 - an operation still endorsed by William Halsted a century later. ${ }^{2}$

It was not until the late 1800 s that the true spread and pattern of recurrence of breast cancer was realized. Charles Moore (an English surgeon) published a ground-breaking paper describing his observations that breast cancer recurrences had a centrifugal spread from their original site. Moore advocated the routine removal of the involved breast as well as "diseased glands", and the principles of his research are still followed today. With the advent of microscopes and advances in pathology, it became clear that it was not possible to know whether axillary glands were involved without a full histological analysis; therefore, Sir D'arcy Power in 1934 stated that they could never be assumed to be normal. ${ }^{2}$

As the era of routine axillary dissection followed, breast cancer saw a significant reduction in local recurrence rates; however, little impact had been achieved on surgical mortality and morbidity. After a brief period of "super-radical" mastectomy, which included entering the thoracic cavity (to remove internal mammary nodes) and even upper limb amputation, the procedure's climbing mortality rate $(12.5 \%)$ led to its abandonment while surgeons reevaluated the efficacy of such radical surgery. ${ }^{2}$

In the mid-1980s, the benefit of a more "conservative" axillary lymph node dissection (ALND) became recognized as an effective tool both for staging and prognosis in treatment of patients with breast cancer. With the progression of oncological treatment, ALND also helped guide the adjuvant management of surgical patients. The significant morbidity of ALND, in particular lymphedema (up to $28 \%$ of patients dependent on criteria used), demonstrated progress was still required. ${ }^{4}$ This was never more evident than with the introduction of national screening programs, where more nodenegative breast cancers were diagnosed than ever before. It was time for surgeons to turn to less invasive options in the management of the axilla of patients with breast cancer.

\section{Sentinel lymph node biopsy in breast cancer}

The sentinel node technique evolved as an outgrowth of Morton et al's work, mapping the drainage patterns of cutaneous melanoma with lymphoscintigraphy in the mid-1980s., ${ }^{5,42}$ Sentinel lymph node biopsy (SLNB), as a concept, entered breast cancer care in 1994, when Giuliano et al reported the first results of SLNB alone at first surgery in patients with breast cancer. In their report, blue dye identified the sentinel lymph node (SLN) in $65 \%$ of 173 cases, with $96 \%$ accuracy. ${ }^{6}$

Over 10 years later in 2006 , Kim et al conducted a systematic review and meta-analysis including over 8,000 patients with breast cancer. ${ }^{7}$ Overall, the SLN localization rate was $96.4 \%$, and the estimated false negative rate was $7.0 \%$. Techniques for localization of the SLN evolved, and blue dye combined with radioisotope emerged as the most effective tracing agents to identify the SLN(s) in breast cancer, superior to single-agent localization alone (odds ratio $[\mathrm{OR}]=2.03$ ). ${ }^{8}$

Identifying and removing only the first lymph nodes to drain the breast cancer ensured that more than $70 \%$ of patients with early breast cancer without lymph node metastasis at diagnosis were spared a full axillary dissection and its associated morbidity and healthcare cost. ${ }^{9}$ Furthermore, patients undergoing SLNB alone recover quicker, have a reduced incidence of lymphedema and nerve injury, and an improved quality of life, compared to patients undergoing routine axillary node clearance. ${ }^{10}$

The SLNB is, today, considered one of the most significant breakthroughs of breast cancer surgery in recent times. The National Institute for Health and Care Excellence (UK) revised its guidelines in 2009 to include routine SLNB for patients with breast cancer and clinically benign axillae. ${ }^{11}$

\section{Intraoperative SLN analysis}

Dual localization sentinel node surgery became standard practice for the surgical staging and prognostic planning of treatment for patients with breast cancer. ${ }^{10}$ Surgeons next turned to finding an accurate, timely, and reliable intraoperative assessment tool that would obviate the need for a second operation to treat the axilla in SLN-positive patients, thereby expediting patient progression to adjuvant oncological treatment. ${ }^{12}$ Moreover, secondary analysis of the ALMANAC study proved if patients were to undergo a one-step procedure with intraoperative SLN analysis they had a significantly shorter length of hospital stay, axillary operative time, and no difference in surgical complication rate. ${ }^{12}$

Prior to one-step nucleic acid amplification (OSNA), SLN analysis could be conducted via hematoxylin and eosin (H\&E)-based histopathological examination of frozen section or cytological assessment of touch imprints, followed by definitive postoperative histopathological examination of permanent sections. However, there were shortcomings in these techniques that prevented definitive SLN analysis on a large scale. The sensitivity of touch imprints varied from $33 \%$ to $73 \%$, and often presented false-negative results in the 
presence of micro-metastases and invasive lobular carcinomatous disease. ${ }^{13}$ Histopathological analysis is an expensive method of SLN analysis, requiring a consultant pathologist on hand to analyze the specimen, thereby making interpretation operator dependent. The sensitivity of histopathological analysis varies in the literature from $54 \%$ to $74 \%$, and the process of cutting a frozen section (alternate slices of the node analyzed) causes permanent tissue loss and theoretical under-staging of sentinel node disease, the frequency of which is impossible to determine through direct comparison with alternative methods. ${ }^{13}$

To overcome these shortcomings, molecular biological methods based on quantitative reverse transcription-PCR (qRT-PCR) were studied extensively to objectively and efficiently detect metastatic lymph node disease in patients with breast cancer. A qRT-PCR assay with multiple mRNA markers, including cytokeratin 19 (CK19), trefoil factor 3 (p1B), epithelial glycoprotein 2 (EGP2), and small breast epithelial mucin (SBEM), resulted in a $10 \%$ improvement compared to histopathological methods. ${ }^{14}$ In addition, there was research into mRNA markers of CK19 and mammaglobin 1 (MGB1), which were found to have accuracy approaching that of histopathology; however, concerns were raised in pseudogene formation causing false-positive results when using single markers only via the qRT-PCR amplification process. ${ }^{15}$

In light of this, Tsujimoto et al in Japan reported an innovative OSNA assay. This consisted of solubilizing the whole lymph node, followed by reverse transcription loop-mediated isothermal amplification (RT-LAMP) of a target mRNA CK19. The new method of gene amplification had a high specificity for target mRNA in the metastatic lymph node, avoiding genomic DNA amplification (or CK19 pseudogene amplification), the CK19 marker is known to be expressed in over $95 \%$ of all breast cancers. The OSNA method was the first to provide objective semi-quantitative measurements of the target mRNA in a metastatic lymph node, and initial results were promising. ${ }^{15}$

Tsujimoto et al studied OSNA assay versus three-level histopathology (a superior histopathological method to the routine two-level one). Lymph nodes within this study were halved, using one half for OSNA analysis and the other half for histopathological evaluation. The concordance rate of OSNA and histopathological analysis were reported to be $98.2 \% .{ }^{15}$ Furthermore, the OSNA assay discriminated macro-metastases from micro-metastases using cutoff values of 5,000 and 250 copies $/ \mu \mathrm{L}$, respectively. In addition, no false-positive results were demonstrated in their initial trial of 353 lymph nodes, suggesting an extremely low false-positive rate. ${ }^{15}$ Sensitivity studies followed (Table 1). Moreover, the median time for OSNA analysis was uniquely low; a single SLN processing took 32 minutes, and 42 minutes for two sentinel nodes. ${ }^{16,21}$

Further multicenter analysis by Wang et al of 552 patients compared intraoperative analysis of SLNs between OSNA, frozen section, and imprint cytology. A total of 1,188 SLNs were studied. OSNA was found to be superior to frozen section for assay sensitivity (77.6\% vs $69.7 \%)$ and was significantly superior to imprint cytology (83.6\% vs $76.2 \%)$. For nodes with micro-metastases, the sensitivity of the assay was higher than that with frozen section and was again significantly higher than with touch imprint cytology. The median turnaround time of the OSNA assay was 37 minutes, and it was deemed accurate and rapid in the analysis of intraoperative lymph nodes. ${ }^{19}$

Multiple studies have since compared OSNA and histopathological examination. It is now accepted that OSNA is at least equivalent to pathological examination in detecting lymph node metastasis; however, $100 \%$ concordance rates between the two techniques will never be demonstrated due to tumor allocation bias. Advantages of OSNA over histopathology, other than in its increased sensitivity in detecting

Table I Summary of data comparing OSNA with routine histopathological examination in detecting positive SLN

\begin{tabular}{|c|c|c|c|c|c|c|c|}
\hline Study & Year & $\begin{array}{l}\text { Number of } \\
\text { lymph nodes }\end{array}$ & Sensitivity (\%) & Specificity (\%) & $\begin{array}{l}\text { Positive predictive } \\
\text { value (\%) }\end{array}$ & $\begin{array}{l}\text { Negative } \\
\text { predictive } \\
\text { value (\%) }\end{array}$ & $\begin{array}{l}\text { Concordance } \\
\text { rate }(\%)\end{array}$ \\
\hline Tsujimoto et al $\left.\right|^{15}$ & 2007 & 325 & 95.8 & 98.6 & 91.5 & 99.3 & 98.2 \\
\hline Tamaki et al ${ }^{17}$ & 2009 & 450 & 87.5 & 94.1 & 76.1 & 97.2 & 92.9 \\
\hline Feldman et $\mathrm{al}^{18}$ & 2011 & 1044 & 77.5 & 95.8 & 73.8 & 96.6 & 93.4 \\
\hline Snook et al ${ }^{16}$ & 2011 & 395 & 91.7 & 96.9 & 86.8 & 98.1 & 95.9 \\
\hline Wang et al ${ }^{19}$ & 2012 & 1188 & 83.7 & 92.9 & 96.8 & 69.1 & 91.4 \\
\hline Buglioni et $\mathrm{al}^{20}$ & 2013 & 903 & 92.6 & 96.1 & 86.1 & 98.0 & 95.3 \\
\hline Banerjee et $\mathrm{a}^{21}$ & 2014 & 268 & 95.1 & 95.6 & 80.0 & 99.1 & 95.5 \\
\hline \multirow[t]{2}{*}{ Chaudhry et al ${ }^{22}$} & 2014 & 166 & 92.8 & 93.7 & 43.4 & 99.2 & 89.2 \\
\hline & & & & & macro-metastases only & & \\
\hline
\end{tabular}

Abbreviations: OSNA, one-step nucleic acid amplification; SLN, sentinel lymph node. 
micro-metastases, ${ }^{23}$ is its demonstration of semi-quantitative data on tumor volume in the entire lymph node rapidly and cost-effectively, with little input from pathologists and less interobserver variability.

OSNA is now validated as an accurate and reliable tool, evidenced by the National Institute for Health and Care Excellence (NICE) approving its use in staging the axilla of patients with breast cancer in $2013 .{ }^{24}$ It is the most widely used axillary staging method in the United Kingdom, and over 200 hospitals in Europe and Asia as well as 102 hospitals in Japan use one-step nucleic acid amplification assay routinely. ${ }^{25}$

\section{Cost-effectiveness of OSNA}

The NICE has previously evaluated the cost-effectiveness of OSNA, and made recommendations in their guidelines based on five studies. One of these studies reported that OSNA had a 0.1 quality-adjusted life year (QALYS) less than the long-term gold standard. ${ }^{25}$ However, this assumed sensitivity and specificity of the gold standard was correct. Therefore, progression-free survival in OSNA versus the gold standard cohorts was lacking, preventing us from evaluating whether OSNA was truly inferior to the gold standard when interpreting cost-effectiveness.

Saruta and Puig-Junoy set out to answer this question, measuring the budget impact in Japanese patients with breast cancer after the introduction of OSNA while assessing the certainty of the results. They evaluated 70,626 patients with newly diagnosed breast cancer undergoing SLN biopsy during a 5-year follow-up period, using a total tumor load (TTL) cut-off for ALND of 15,000 copies/ $\mu \mathrm{L}$. The introduction of OSNA improved the diagnostic accuracy and, in all cases, the test scenarios revealed lower costs as compared with the reference (or gold standard) scenario over a 5-year period. Saruta and Puig-Junoy discovered a reduction of \$346 per patient in healthcare costs when using intraoperative OSNA, as confirmed by sensitivity analysis. ${ }^{25}$

\section{Defining role of OSNA}

The last 12 months have seen practice-changing research come to the forefront of axillary management for patients with breast cancer. The AMAROS, IBCSG 12-01, and ACOSOG Z0011 trials have together shown that patients with T1-3 breast cancer with limited micro- or macro-metastatic SLN involvement (no more than two metastasis-positive sentinel nodes) have similar local recurrence-free survival and overall survival regardless of whether they undergo axillary node clearance, provided those who do not undergo axillary dissec- tion receive both whole-breast irradiation and systemic adjuvant treatment. ${ }^{26-28)}$ This new evidence promises significantly lower morbidity of lymphedema, nerve injury (including chronic pain), and reduced movement of the arm that are currently seen twice as commonly with axillary node dissection versus sentinel node biopsy and adjuvant irradiation of the whole breast. ${ }^{26}$ However, quality of life appears equivalent. ${ }^{26}$

Great lengths have gone toward identifying cohorts of patients with positive sentinel nodes who can safely avoid routine axillary node dissection and, therefore, do not require intraoperative sentinel node analysis. Multiple randomized trials over the last 12 months have attempted to clarify this question.

The International Breast Cancer Study Group (IBCSG) trial 23-01 that was the earliest randomized controlled trial (RCT) to report on this topic, enrolled patients over a 9 -year period, and completed in 2010. The study randomized patients with micro-metastasis or isolated tumor cells (ITCs) within the SLN, (based on histology results [H\&E staining]), to axillary lymph node dissection (ALND) versus no axillary lymph node dissection. The aim of this prospective randomized clinical trial was to evaluate the impact of avoiding ALND on long-term patient outcomes, and occurred during a time when surgeons were already changing their practice in undertaking ALND for micro-metastatic SLN disease less frequently. The results of the study demonstrated no difference in both study arms of patients in 5-year disease-free survival and locoregional recurrence rates.

The IBCSG 23-01 originally aimed to enroll 1,960 patients; ${ }^{27}$ however, enrolment stopped prematurely at 933 patients due to the select inclusion criteria. The target group of patients required small, estrogen receptor (ER)-positive breast cancers undergoing breast conservation with planned adjuvant whole-breast irradiation and very low-volume axillary disease. Only $14 \%$ of the 6,681 registered patients for this study were included. ${ }^{27}$ The study was underpowered.

The patient cohort of the IBCSG 23-01 had very good prognosis breast cancers, both clinically and histologically. Isolated tumor cells were included under the umbrella of "micrometastasis", and, almost certainly as an impact of this, nearly $70 \%$ of patients included had SLN tumor size of less than 1 $\mathrm{mm}$, or "mini-metastasis" as it has become known. This information, along with the study's limited follow-up of 5 years, has led surgeons to be cautious in its clinical interpretation.

Original data from the National Surgical Adjuvant Breast and Bowel project (NSABP) B-32 trial found, of the 5,611 women with operable clinically N0 invasive breast cancer, carrying out SLNB with ALND had no significant impact on 
the axillary recurrence rate compared to those who underwent sentinel node biopsy alone; however, 10-year follow-up data demonstrated conflicting results. Upon retrospective central pathology review, 616 women included in this trial had occult, positive axillary nodal disease -430 patients had isolated tumor cell clusters, 172 had micro-metastases, and 14 had macro-metastases. Comparisons between the two cohorts of women with and without occult axillary disease demonstrated a $2.8 \%$ absolute difference in overall survival at 10 years (HR $1.25 ; p=0.08)$ and $4.1 \%$ absolute difference in disease-free survival (HR 1.24; $p=0.018$ ) in favor of SLNB with ALND. ${ }^{29}$ These data added caution in counseling patients, particularly younger women with otherwise curable tumors, that the long-term impact of limited axillary surgery for low-volume axillary disease was still relatively unknown.

The ACOSOG Z0011 trial enrolled patients with clinical $\mathrm{T} 1$ or $\mathrm{T} 2$ breast cancers that had clinically benign axillae, undergoing breast-conserving surgery with micro- or macro-metastases in two or less SLNs found intraoperatively. Patients were randomized to either completion axillary lymph node clearance or observation. All patients received adjuvant whole-breast irradiation. Results at a median of 6.3 years found no significant difference between the two study arms in terms of regional recurrence, and 5-year overall and disease-free survival was similar between the two arms. Further results were published last year at 9.25 years follow-up. At this point, Giuliano et al found $0.5 \%$ of ALND patients had local recurrence in the ipsilateral axilla versus $1.5 \%$ of SLND-only patients. The results were not statistically significant, as were the remaining endpoints. ${ }^{28}$

The ACOSOG Z0011 trial similarly came under criticism for being under-powered as it was designed to enroll 1,900 but accrued less than 900 (due to slow accrual rate and low event rate). Moreover, the study was criticized for recruiting women with only low-risk breast cancers with almost $70 \%$ T1 tumors, of which $83 \%$ were ER positive. Ninety-seven percent of patients received adjuvant systemic therapy. In addition, there was a discrepancy between the number of patients in the two arms; a statistically significant higher proportion of patients were enrolled to the SLN group with micro-metastasis versus the ALND (44.8\% vs 37.5\%). Further to this, there were concerns with regards to compliance with the study's protocol for whole-breast irradiation using tangential fields only; prohibiting third-field-directed nodal treatment. This was looked into by Jagsi et al, ${ }^{30}$ who noted that, of the 891 patients included in the Z0011 study, only 228 had details of the radiotherapy (RT) regimens documented. Of these patients, 43 (18.9\%) received prohibited directed regional nodal RT using three fields or more: 22 in the ALND arm and 21 in the SLND arm. Those receiving directed nodal RT had greater nodal involvement $(p<0.001)$ than those who did not. The impact of such targeted treatment is unclear; however, it has the potential to dampen any difference between the two arms.

In 2016, the American Society of Clinical Oncology (ASCO) updated their guidelines for SLNB and ALND indicating that patients with micro- and macro-metastases may avoid ALND in response to the results of the IBCSG 23-01 trial along with the American College of Surgeons Group (ACOSOG) Z0011 trial.

However, a trial that was generalizable to a greater population of patients was needed, and hope came in the form of the EORTC 10981-2203 AMAROS trial (After Mapping of the Axilla: Radiotherapy or Surgery?). This was the first prospective trial to randomize patients with small $\mathrm{T} 1$ or T2 breast cancers with no palpable lymphadenopathy and a positive SLN result to either completion ALND or adjuvant axillary irradiation. Axillary irradiation targeted levels I-III as well as the medial supraclavicular region. The AMAROS reported local axillary recurrence rates of $0.43 \%$ at 5 years in the ALND arm and $1.19 \%$ in the SLN and axillary irradiation arm (not statistically significant). In addition, there were no significant differences in disease-free and overall survival between the two arms.

The benefit of SLNB and axillary RT over ALND reported from the AMAROS trial, which was again, underpowered, was a significantly lower incidence of lymphedema. There was, however, no statistically significant or clinically relevant differences found in quality of life between the two groups for the selected scales of arm symptoms, pain, or body image.

Only $9 \%$ of the patients included in the AMAROS trial underwent mastectomy, and $15 \%$ of patients had four or more proven involved axially lymph nodes. Therefore, the numbers are generally too small to have reliable data on treatment of this cohort of patients. In addition, there remains a significant proportion of patients who may not meet the AMAROS trial criteria due to tumor size, tumor biology, extranodal disease, undergoing neoadjuvant chemotherapy (NAC), or wishing to avoid adjuvant RT.

While the AMAROS trial undoubtedly represents a landmark in potentially changing clinical practice, longer follow-up is still required. The continued trepidation of oncologists and breast surgeons is hoped to be addressed by the ongoing clinical trials including POSNOC (Positive Sentinel Node: Adjuvant Therapy Alone Versus Adjuvant Therapy Plus Clearance or Axillary Radiotherapy).

POSNOC, a prospective multicenter RCT, aims to randomize patients with one or two macro-metastases within 
their SLNs to standard adjuvant therapy or adjuvant therapy with ALND or targeted axillary (levels I-III) RT. It is the first trial of its kind to randomize patients to targeted RT of the axilla, and is also ground breaking in allocating patients with two macro-metastases to no targeted axillary treatment at all. One hypothesis of the trial is that low-volume axillary disease is adequately treated with systemic therapy only in preventing axillary recurrence. While enrollment has begun for this trial, its predicted end date is not until December 2023. ${ }^{31}$

Despite this building evidence, there remains a cohort of patients where axillary dissection has a very important role in axillary management for SLN-positive disease, especially those patients undergoing mastectomy. All of the patients of the IBCSG 23-01 trial and over 90\% of those in the AMAROS trial underwent breast-conservation surgery. There is a paucity of high-level evidence proving whether mastectomy patients and those with four or more positive lymph nodes can safely avoid axillary dissection, some of whom would not undergo irradiation of the chest wall.

A further subset of patients are those with T4 tumors, breast cancers with poor prognostic markers (such as lymphovascular invasion, high Ki67 growth fraction, and grade 3 status), and clinical or radiological evidence of four or more lymph nodes with metastatic involvement. This subset of patients have a higher incidence of non-SLN disease. Avoiding ALND in this cohort of patients prevents targeted supraclavicular fossa irradiation and full computed tomography (CT) staging should ALND have otherwise demonstrated high volume metastatic axillary disease.

SLNB with intraoperative analysis with or without ALND, therefore, continues to be the clinical standard of practice for both patients undergoing mastectomy and those with poor prognostic tumors.

A subset of patients who have been the cause for much debate since intraoperative lymph node analysis came into common practice are those who have undergone NAC. Following NAC, lymph nodes frequently show significant histological change due to stromal fibrosis and elastosis, thereby distorting ready identification of residual, viable, metastatic tumor cells. OSNA measures CK19 derived from viable tumor cells and, thus, should accurately assess changes more readily than pathological examination. Studies examining OSNA in NAC patients are few, and small in sample size. While some report accuracy, sensitivity, and specificity rates of OSNA of $90.9 \%, 88.9 \%$, and $93.3 \%$, respectively, ${ }^{32}$ others report OSNA sensitivity to be significantly lower in SLNs following NAC. Takamoto et al reported a drop, following NAC, in OSNA sensitivity from $95.1 \%$ to $87.8 \%$ in non-sentinel nodes versus SLNs. ${ }^{33}$ There is a suggestion that CK19 mRNA expression in primary breast tumors is reduced following NAC ${ }^{33}$ and, therefore, this may correspond to reduced expression in the SLN; however, Vieites et al discovered evidence to the contrary. Their evidence comparing breast tumor and node samples before and after NAC found that the CK19 mRNA was preserved. ${ }^{34}$ Parada et al had similar findings last year from an institutional study of 50 patients undergoing SLN analysis and OSNA following NAC. ${ }^{35}$ Of those patients with "positive SLN's" (ITC as well as micro- and macro-metastases), all were subject to ALND. Only two cases with macro-metastases (TTL SLN: 27,000 and 2,100,000 copies/ $\mu L$ ) showed additional positive non-SLNs on ALND.

It remains unclear whether CK19 mRNA expression and, indeed, OSNA sensitivity are affected by NAC; however, if CK19 expression is a reflection of the viability of tumor cells, it may prove a more accurate marker to assess effectiveness of chemotherapy and may be, therefore, a more reliable prognostic marker for patients treated with NAC. Further evidence for this cohort of patients is required, and it is unlikely we will have a higher level of knowledge on this in the near future, evidenced by NAC being an exclusion criteria for the POSNOC trial.

For the moment, intraoperative SLN analysis cannot be safely relied upon for patients with proven pre-NAC metastatic disease (in more than two nodes) and, therefore, these patients require ALND as standard practice.

Combining the best evidence discussed here with our own clinical experience using OSNA at one of the largest breast cancer units in the country, we propose an algorithm for the role of OSNA in managing patients with newly diagnosed breast cancer (Figure 1). From reviewing the literature, this appears to be the first clinical algorithm of its kind for the use of OSNA for patients with breast cancer.

\section{Ongoing trials of OSNA}

As the use of intraoperative sentinel node analysis falls, attention has begun to focus on redefining the role of OSNA to utilize the TTL (total CK19 mRNA copy number of all sentinel nodes) as a guide to non-sentinel node involvement and prognosis.

OSNA may prove a rational method in decision-making for omitting axillary lymph node clearance in the presence of positive sentinel nodes, as it is a quantitative technique that can provide additional markers beyond the 5,000 copies $/ \mu \mathrm{L}$ macro-metastasis cut-off. In the last 3 years, focus has been on the relationship between CK19 mRNA copy numbers as a molecular measure of tumor load in the sentinel node and its predictive value of non-sentinel node involvement in the axilla. Multiple studies have found that, as the tumor load of the total sentinel node(s) count rise, the predictive value of non-sentinel node positivity increases. Cut-off thresholds 


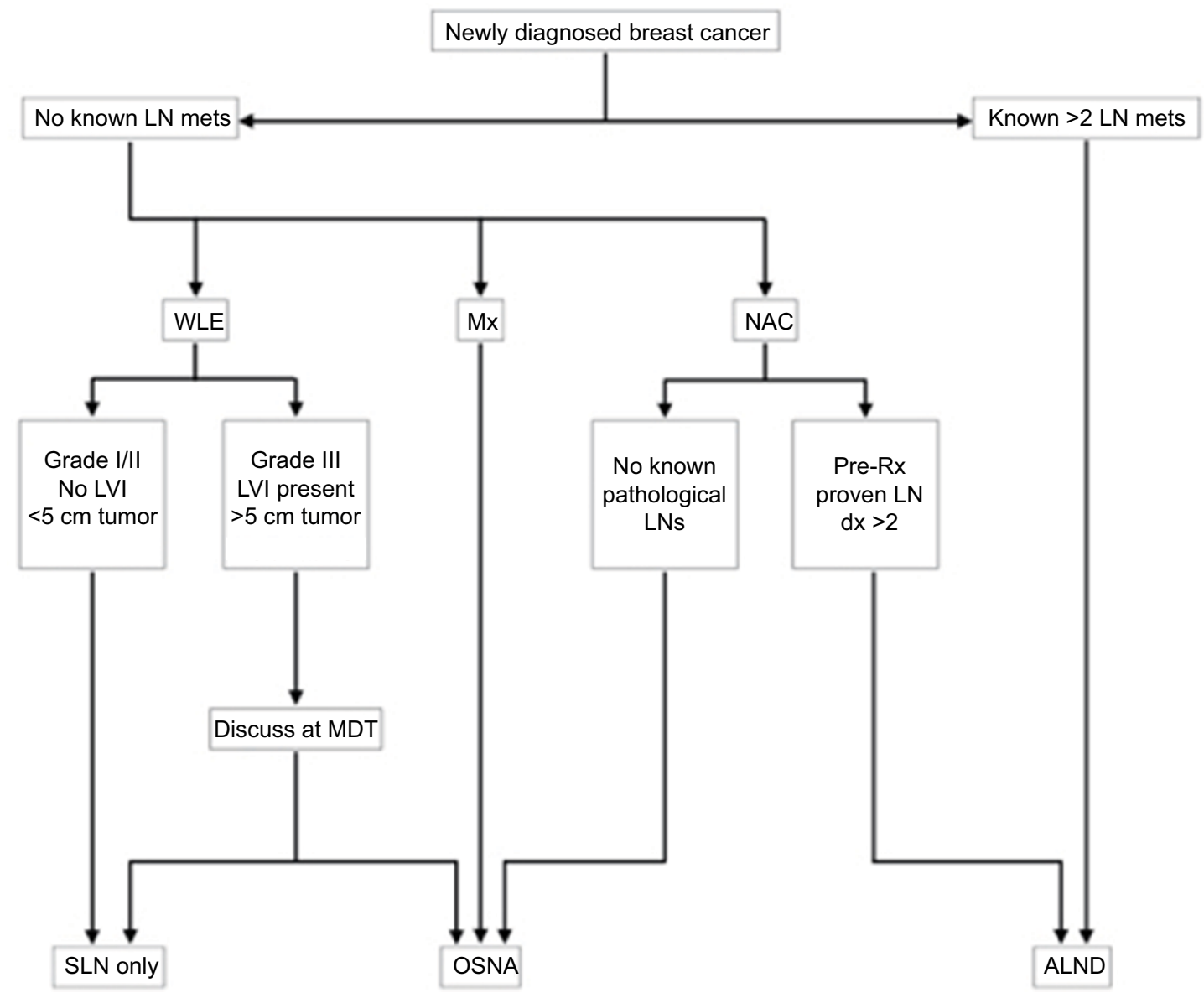

Figure I Best evidence management of the axilla in patients with newly diagnosed breast cancer.

Abbreviations: LN, lymph node; mets, metastases; WLE, wide local excision; Mx, mastectomy; NAC, neoadjuvant chemotherapy; LVI, lymphovascular invasion; Pre-Rx, pre-therapy; MDT, multidisciplinary team meeting; ALND, axillary lymph node dissection; OSNA, one-step nucleic acid amplification; SLN, sentinel lymph node; dx, disease.

for detecting more than one additional positive non-sentinel nodes have been suggested to be approximately 7,700 mRNA copies $/ \mu \mathrm{L}$, but a threshold this high also results in an unacceptably high false-negative rate $(17.4 \%){ }^{36}$

Histopathological assessment of the SLN biopsy is capable of predicting the likelihood of non-sentinel node involvement by the size of metastasis, with a predictive accuracy of $50 \%$ for metastases greater than $2 \mathrm{~mm}$. It is, therefore, not currently used clinically to predict the number of nodes involved. In contrast, however, the total copy number for all positive sentinel nodes using OSNA, referred to as the TTL has been strongly suggested to predict the number of nodes involved. ${ }^{37}$

Beyond this, it is also important to take into account the role of tumor biology in axillary disease. Factors found to be associated with non-sentinel node positivity include tumor grade, sentinel node involvement pattern (isolated tumor cells versus micro- or macro-metastasis), and lymphovascular invasion of the primary tumor. ${ }^{37}$ Therefore, would a model that includes TTL and tumor-related factors provide a patient-specific risk assessment?
Milner et al collected 856 SLNs analyzed using OSNA from patients who were clinically node negative in 2015 . They aimed to clarify risk factors for non-SLN involvement upon axillary node dissection. As expected, $25.3 \%$ of these SLNs had metastatic disease, and patients underwent ALND. Multivariate analysis was conducted, and significant risk factors for non-SLN involvement were SLN macrometastasis ( $\mathrm{CK}$ count $>5,000$ copies $/ \mu \mathrm{L})$, multiple allpositive SLNs (over variable positive SLNs), and patients undergoing mastectomy. ${ }^{38}$ The importance of non-SLN involvement continues to be relevant to these patients who require targeted supraclavicular fossa irradiation and formal CT staging.

The suggestion that OSNA could predict non-SLN positivity based on factors other than simply a positive or negative count has led others to explore the potential for OSNA's quantitative nature in identifying patients with positive non-SLNs further.

A series of studies have recently been carried out focusing on TTL calculation in the form of objective quantitative results. These studies have suggested that TTL may predict 
the axillary status of patients with breast cancer, thereby avoiding unnecessary ALND.

Deambrogio et al reviewed SLN TTL from over 1,000 patients in 2014, and found that those with a TTL of greater than 7,700 all had involved non-sentinel nodes, and one in nine patients whose TTL ranged between 5,000 and 7,700 had involved non-sentinel nodes. ${ }^{36}$

This guidance for intraoperative decision making is being expanded further to develop a nomogram using OSNA TTL as part of a risk prediction tool, dictating who should have axillary lymph node dissection and who should not. A nomogram technique using multivariate analysis of tumor size, number of affected SLN's, Her2 expression, lymphovascular invasion, as well as TTL was developed by Deambrogio et al. The overall predictive accuracy of the nomogram was $0.7552 .{ }^{39}$

Kubota et al examined 134 patients undergoing OSNA analysis of SLNs followed by ALND, aiming to identify factors associated with four or more lymph node metastases via multivariate analysis. ${ }^{40}$ They examined pathological factors including tumor size, histological grade, lymphovascular invasion, and histology, none of which were found to be correlated with four or more lymph node metastases. Many of these factors, which have been associated with non-sentinel node positivity in conflicting studies, are unreliable as an intraoperative decisionmaking tool due to tumor characteristics (eg, lymphovascular invasion and histological grade) changing between preoperative core biopsy and postoperative histopathological analysis. In Kubota et al's study, the single significant factor associated with four or more lymph node metastases was a TTL greater than 5,400 copies/ $\mu \mathrm{L}$. This study suggested the quantitative OSNA tool may be used beyond its current limits, as an objective tool for intraoperative decision making with regard to the omission of ALND in specified SLN-positive patients, independent of the number of positive sentinel nodes. ${ }^{40}$

Peg et al attempted to utilize the TTL as a prognostic marker of survival in early breast cancer. Their study of 950 patients between 2009 and 2010 found that TTL significantly correlates with disease-free, local recurrence-free, and overall survival. This correlation was independent of axillary staging, patient age, and tumor characteristics (size, grade, and lymphovascular invasion). They identified a low-risk group of women (TTL less than 2,500 mRNA copies/ $\mu \mathrm{L}$ ) and a high-risk group (TTL greater than 2,500 mRNA copies/ $\mu \mathrm{L}$ ). These data suggest TTL to be a potential prognostic staging tool for the axilla that is lost in patients with early breast cancer who no longer undergo ALND. ${ }^{41}$

The TTL as a predictive tool clearly requires further validation studies before its use in routine practice. However, with hopes to minimize morbidity associated with surgical axillary node clearance, the potential for OSNA to provide a higher predictive value for non-sentinel node involvement will continue to be of interest.

\section{Conclusion}

We have reviewed the evidence base for the role of intraoperative SLN analysis, highlighting the defining role of OSNA over the past decade. We believe OSNA remains a valuable predictive tool in avoiding a second operation for patients undergoing mastectomy as primary surgery and for those with tumors with poor prognostic markers, to enable full treatment and staging of these patients. We propose a clinical algorithm demonstrating OSNA's role in today's practice. Further validation studies are required for its use in patients following NAC, as well as its use as part of a TTL analysis tool.

\section{Disclosure}

The authors report no conflicts of interest in this work.

\section{References}

1. World Health Organization. Breast Cancer: prevention and control 2017. Available from: http://who.int/cancer/detection/breastcancer/en/. Accessed July 15, 2017

2. Rayter Z, Mansi J. Medical Therapy of Breast Cancer. Cambridge: Cambridge University Press; 2003.

3. Fund WCR. Breast cancer: incidence 2017. Available from: https:// www.wcrf-uk.org/uk/preventing-cancer/cancer-preventability-statistics/ breast-cancer. Accessed July 15, 2017.

4. DiSipio T, Rye S, Newman B, Hayes S. Incidence of unilateral arm lymphoedema after breast cancer: a systematic review and metaanalysis. Lancet Oncol. 2013;14(6):500-515.

5. Tanis PJ, Nieweg OE, Valdés Olmos RA, Th Rutgers EJ, Kroon BB. History of sentinel node and validation of the technique. Breast Cancer Res. 2001;3(2):109-112.

6. Giuliano AE, Kirgan DM, Guenther JM, Morton DL. Lymphatic mapping and sentinel lymphadenectomy for breast cancer. Ann Surg. 1994;220(3):391-398; discussion 398-401.

7. Kim J, Giuliano AE, Turner RR, et al. Lymphatic mapping establishes the role of BRAF gene mutation in papillary thyroid carcinoma. Ann Surg. 2006;244(5):799-804.

8. He PS, Li F, Li GH, Guo C, Chen TJ. The combination of blue dye and radioisotope versus radioisotope alone during sentinel lymph node biopsy for breast cancer: a systematic review. BMC Cancer. 2016;16:107.

9. Cancer Research UK. Breast cancer incidence (invasive) statistics 2017. Available from: http://www.cancerresearchuk.org/health-professional/ cancer-statistics/statistics-by-cancer-type/breast-cancer/incidenceinvasive\#heading-Three. Accessed July 15, 2017.

10. Kim T, Giuliano AE, Lyman GH. Lymphatic mapping and sentinel lymph node biopsy in early-stage breast carcinoma: a metaanalysis. Cancer. 2006;106(1):4-16.

11. NICE National Institute for Health and Care Excellence. Everything NICE says on a topic in an interactive flowchart 2009. Available from: https://pathways.nice.org.uk. Accessed July 15, 2017.

12. Fortunato L, Amini M, Farina M, et al. [Extemporaneous examination of the sentinel lymph node in breast cancer: is the glass half full or half empty?]. Tumori. 2003;89(4 Suppl):192-193. Italian.

13. Layfield DM, Agrawal A, Roche H, Cutress RI. Intraoperative assessment of sentinel lymph nodes in breast cancer. Br J Surg. 2011;98(1):4-17. 
14. Weigelt B, Verduijn P, Bosma AJ, Rutgers EJ, Peterse HL, van't Veer LJ. by multiple mRNA markers. Br J Cancer. 2004;90(8):1531-1537.

15. Tsujimoto M, Nakabayashi K, Yoshidome K, et al. One-step nucleic acid amplification for intraoperative detection of lymph node metastasis in breast cancer patients. Clin Cancer Res. 2007;13(16):4807-4816.

16. Snook KL, Layer GT, Jackson PA, et al; OSNA Study Group. Multicentre evaluation of intraoperative molecular analysis of sentinel lymph nodes in breast carcinoma. Br J Surg. 2011;98(4):527-535.

17. Tamaki Y, Akiyama F, Iwase T, et al. Molecular detection of lymph node metastases in breast cancer patients: results of a multicenter trial using the one-step nucleic acid amplification assay. Clin Cancer Res. 2009;15(8):2879-2884.

18. Feldman S, Krishnamurthy S, Gillanders W, et al; US One Step Nucleic Acid Amplification Clinical Study Group. A novel automated assay for the rapid identification of metastatic breast carcinoma in sentinel lymph nodes. Cancer. 2011;117(12):2599-2607.

19. Wang YS, Ou-yang T, Wu J, et al. Comparative study of one-step nucleic intraoperative assessment of breast sentinel lymph node in Chinese patients. Cancer Sci. 2012;103(11):1989-1993.

20. Buglioni S, Di Filippo F, Terrenato I, et al. Quantitative molecular analysis of sentinel lymph node may be predictive of axillary node status in breast cancer classified by molecular subtypes. PLoS One. 2013;8(3):e58823.

21. Banerjee SM, Michalopoulos NV, Williams NR, et al. Detailed evaluation of one step nucleic acid (OSNA) molecular assay for intraoperative diagnosis of sentinel lymph node metastasis and prediction of non-sentinel nodal involvement: experience from a London teaching hospital. Breast. 2014;23(4):378-384.

22. Chaudhry A, Williams S, Cook J, et al. The real-time intra-operative evaluation of sentinel lymph nodes in breast cancer patients using One Step Nucleic Acid Amplification (OSNA) and implications for clinical decision-making. Eur J Surg Oncol. 2014;40(2):150-157.

23. Tiernan JP, Verghese ET, Nair A, et al. Systematic review and metaanalysis of cytokeratin 19-based one-step nucleic acid amplification versus histopathology for sentinel lymph node assessment in breast cancer. Br J Surg. 2014;101(4):298-306.

24. Rayter Z. In defence of one step nucleic acid amplification for intraoperative assessment of axillary lymph nodes in breast cancer. $B M J$ 2015;350:h716.

25. Saruta Y, Puig-Junoy J. Cost and budget impact analysis of an accurate intraoperative sentinel lymph node diagnosis for breast cancer metastasis. Appl Health Econ Health Policy. 2016;14(3):323-335.

26. Donker M, van Tienhoven G, Straver ME, et al. Radiotherapy or surgery of the axilla after a positive sentinel node in breast cancer (EORTC 10981-22023 AMAROS): a randomised, multicentre, open-label, phase 3 non-inferiority trial. Lancet Oncol. 2014;15(12):1303-1310.

27. Galimberti V, Cole BF, Zurrida S, et al; International Breast Cancer Study Group Trial 23-01 investigators. Axillary dissection versus no axillary dissection in patients with sentinel-node micrometastases (IBCSG 23-01): a phase 3 randomised controlled trial. Lancet Oncol. 2013;14(4):297-305.

28. Giuliano AE, Ballman K, McCall L, et al. Locoregional recurrence after sentinel lymph node dissection with or without axillary dissection in patients with sentinel lymph node metastases: long-term follow-up from the American College of Surgeons Oncology Group (Alliance) ACOSOG Z0011 randomized trial. Ann Surg. 2016;264(3):413-420. Detection of metastases in sentinel lymph nodes of breast cancer patients acid amplification assay, frozen section, and touch imprint cytology for

29. Julian TB, Krag DN, Waeaver DL, et al. 10-year follow-up results of occult detected sentinel node disease: NSABP B-32, a randomized phase III clinical trial to compare sentinel node resection (SNR) to conventional axillary dissection $(\mathrm{AD})$ in clinically node-negative breast cancer patients. San Antonio Breast Cancer Symposium; San Antonio; 2013.

30. Jagsi R, Chadha M, Moni J, et al. Radiation field design in the ACOSOG Z0011 (Alliance) Trial. J Clin Oncol. 2014;32(32):3600-3606.

31. Goyal A, Dodwell D. POSNOC: a randomised trial looking at axillary treatment in women with one or two sentinel nodes with macrometastases. Clin Oncol (R Coll Radiol). 2015;27(12):692-695.

32. Osako T, Tsuda H, Horii R, et al. Molecular detection of lymph node metastasis in breast cancer patients treated with preoperative systemic chemotherapy: a prospective multicentre trial using the one-step nucleic acid amplification assay. Br J Cancer. 2013;109(6):1693-1698.

33. Takamoto K, Shimazu K, Naoi Y, et al. One-step nucleic acid amplification assay for detection of axillary lymph node metastases in breast cancer patients treated with neoadjuvant chemotherapy. Ann Surg Oncol. 2016;23(1):78-86.

34. Vieites B, López-García MÁ, Castilla C, et al. CK19 expression in breast tumours and lymph node metastasis after neoadjuvant therapy. Histopathology. 2016;69(2):239-249.

35. Parada D, Peña KB, Riu FF, Aguilar AE, Cohan S. Intraoperative molecular analysis of sentinel lymph nodes following neoadjuvant chemotherapy in patients with clinical node negative breast cancer: an institutional study. Mol Clin Oncol. 2016;5(5):507-510.

36. Deambrogio C, Castellano I, Paganotti A, et al. A new clinical cut-off of cytokeratin 19 mRNA copy number in sentinel lymph node better identifies patients eligible for axillary lymph node dissection in breast cancer. J Clin Pathol. 2014;67(8):702-706.

37. Banerjee SM, Williams NR, Davidson TI, et al. The use of onestep nucleic acid amplification (OSNA) and tumour related factors in the treatment of axillary breast cancer: a predictive model. Eur J Surg Oncol. 2016;42(5):641-649.

38. Milner TD, de Lusignan S, Jones S, et al. Breast cancer metastasis burden in sentinel nodes analysed using one-step nucleic acid amplification predicts axillary nodal status. Breast. 2015;24(5):568-575.

39. Rubio IT, Espinosa-Bravo M, Rodrigo M, et al. Nomogram including the total tumoral load in the sentinel nodes assessed by one-step nucleic acid amplification as a new factor for predicting nonsentinel lymph node metastasis in breast cancer patients. Breast Cancer Res Treat. 2014;147(2):371-380.

40. Kubota M, Komoike Y, Hamada M, et al. One-step nucleic acid amplification assay for intraoperative prediction of advanced axillary lymph node metastases in breast cancer patients with sentinel lymph node metastasis. Mol Clin Oncol. 2016;4(2):173-178.

41. Peg V, Sansano I, Vieites B, et al. Role of total tumour load of sentinel lymph node on survival in early breast cancer patients. Breast. 2017;33:8-13.

42. Morton DL, Wen DR, Wong JH, et al. Technical details of intraoperative lymphatic mapping for early stage melanoma. Arch Surg. 1992;127(4):392-399.

43. National Cancer Intelligence Network. NHS Cancer Screening Programmes. All breast cancer report. A UK analysis of all symptomatic and screen-detected breast cancers diagnosed in 2006. Available from: http://www.ncin.org.uk/view?rid=68. Accessed December 11, 2017.

\section{Dovepress}

\section{Publish your work in this journal}

Breast Cancer - Targets and Therapy is an international, peerreviewed open access journal focusing on breast cancer research, identification of therapeutic targets and the optimal use of preventative and integrated treatment interventions to achieve improved outcomes, enhanced survival and quality of life for the cancer patient.
The manuscript management system is completely online and includes http://www.dovepress.com/testimonials.php to read real quotes from published authors. a very quick and fair peer-review system, which is all easy to use. Visit 